\title{
Clinical outcome of primary small cell carcinoma of the urinary bladder
}

This article was published in the following Dove Press journal:

OncoTargets and Therapy

24 August 2013

Number of times this article has been viewed

\author{
Chen-Pang Hou ${ }^{1,2}$ \\ Yu-Hsiang Lin ${ }^{1,2}$ \\ Chien-Lun Chen ${ }^{1,2}$ \\ Phei-Lang Chang ${ }^{1,2}$ \\ Ke-Hung Tsui ${ }^{1,2}$ \\ 'Department of Urology, Chang \\ Gung Memorial Hospital-Linko, \\ Taiwan, Republic of China; ${ }^{2}$ College \\ of Medicine, Chang Gung University, \\ Taiwan, Republic of China
}

Correspondence: Ke-Hung Tsui

Department of Urology, Chang Gung

Memorial Hospital, Chang Gung

University, \#5 Fu-Shing Street Kwei-Shan,

Tao-Yuan 333, Taiwan, Republic of China

Tel +8863328 I 200 ext 2 I 37

Fax+886227358775

Email khtsui@yahoo.com
Purpose: Primary small cell carcinoma of the urinary bladder is a rare malignant disease. It accounts for less than $1 \%$ of all urinary bladder carcinomas. The purpose of this study is to review the clinical features, the treatment modalities, and the overall survival of these patients. We also compare the clinical outcomes between patients of bladder small cell carcinoma (SCC) and bladder urothelial carcinoma (UC).

Materials and methods: We reviewed the charts of patients with bladder tumors from January 1995 to December 2012 in the Chang Gung Memorial Hospital. A total of 2421 malignant bladder tumor patients were reviewed and there were 18 patients who were diagnosed with primary bladder SCC. The patients' characteristics, including age, gender, smoking history, presented symptoms, tumor size, locations, clinical stages, treatment modalities, pathology appearance, recurrence conditions, and survival conditions were all recorded. We also compared the clinical outcomes and the overall survival rates between patients with bladder SCC and those with UC.

Results: Bladder SCC accounted for about $0.74 \%$ of all bladder malignancies in our institution. The mean age at diagnosis was 70.67 years, and the male-to-female ratio was 2.6:1. Thirteen patients had a history of cigarette smoking. All patients presented with symptoms of gross hematuria, and three of them had bladder tamponade requiring blood clot evacuation by cystoscopy. Only one patient had T1 disease, ten patients had stage III disease, and seven patients had lymph node or distant metastasis (stage IV disease). The mean tumor size was $4.29 \mathrm{~cm}$ in diameter. For the majority (61.11\%) of patients, SCC coexisted with UC components. The average survival time was 10.92 months. Patients with bladder SCC had worse overall survival rates than those of stage III and stage IV bladder UC. Performing radical cystectomy does not significantly improve their overall survival rates. None of the clinicopathologic parameters, including the presence of coexisting nonsmall cell carcinoma component $(P=0.831)$, receiving radical cystectomy $(P=0.194)$, distant metastasis $(P=0.062)$, and gender $(P=0.564)$, were significantly associated with survival.

Conclusion: SCC of the urinary bladder is a rare condition, and standard treatment outlines have not been well established. Most of the presented cases have a very poor prognosis. Prospective, multi-institutional, randomized studies are required to assess better treatment modalities. To the best of our knowledge, this is the largest reported case analysis of primary bladder SCC in a Taiwanese population.

Keywords: bladder, small cell carcinoma, urothelial carcinoma, prognosis

\section{Introduction}

Primary small cell carcinoma of the urinary bladder (SCC) is a rare malignant disease. It accounts for less than $1 \%$ of all urinary bladder carcinomas. ${ }^{1,2}$ The first reported 
case was published in 1981 by Cramer et al. ${ }^{3}$ Since that time, approximately 250 cases have been reported in the literature. ${ }^{4}$ This disease affects mostly elderly male patients who present with gross hematuria, and the prognosis is very poor. ${ }^{5,6}$ However, most of the presented cases were reported in western countries and focused predominantly on the Caucasian population; not many studies focus on Asian populations. We reviewed the charts of Chang Gung Memorial Hospital in Taiwan from January 1995 to December 2012, where a total of 18 cases of primary bladder SCC were recorded. The purpose of this study is to review the clinical features, the treatment modalities, and the overall survival of the patients. We also compare the clinical outcomes between patients with bladder SCC to those with bladder UC. To the best of our knowledge, this is the first reported series of primary bladder $\mathrm{SCC}$ in Taiwanese patients.

\section{Materials and methods}

We reviewed the charts of patients with bladder tumors from January 1995 to December 2012 in the Chang Gung Memorial Hospital. A total of 2421 malignant bladder tumor patients were reviewed. After excluding tumors of other sites with metastasis to the bladder, there were 18 patients who were diagnosed with primary bladder SCC. The tumors fulfilled the criteria established for small cell carcinoma according to the World Health Organization (WHO) classification system. $^{7}$ The patients' characteristics including age, gender, smoking history, presented symptoms, tumor size, locations, clinical stages, treatment modalities, pathology appearance, recurrence conditions, and survival conditions were all recorded. The staging definition for bladder cancer was based on the American Joint Committee on Cancer (AJCC) staging 7th edition. ${ }^{8}$ A Cox proportional hazards model was used to analyze survival outcomes with covariates. We also compared the clinical outcomes and the overall survival rates between patients with bladder SCC and those with bladder UC. KaplanMeier survival curves and Log-rank tests were used to analyze the survival outcomes among the different groups. SPSS 15.0 for windows (IBM Corporation, Armonk, NY, USA) was used for statistical analysis, and $P$-values $\leq 0.05$ were considered statistically significant.

\section{Results}

A total of 2421 malignant bladder tumor patients were reviewed, and there were 18 patients who were diagnosed with primary bladder SCC, which accounted for about $0.74 \%$ of all bladder malignancies in our institution. The 18 patients' characteristics and clinical findings are summarized in Table 1.
Table I Patient characteristics $(\mathrm{n}=18)$

\begin{tabular}{ll}
\hline Characteristics & Number of patients (\%) \\
\hline Age (years) & \\
$<60$ & $1(5.56)$ \\
$60-70$ & $6(33.33)$ \\
$>70$ & $1 \mathrm{I}(61.1 \mathrm{I})$ \\
Gender & \\
Male & $13(72.22)$ \\
Female & $5(27.78)$ \\
Present symptoms & \\
Gross hematuria & $18(100)$ \\
Bladder tamponade & $3(16.67)$ \\
Renal failure & $2(11.11)$ \\
Smoking history & \\
Present & $13(72.22)$ \\
Absent & $5(27.78)$ \\
Initial clinical stage & \\
I & $1(5.56)$ \\
II & $0(0)$ \\
III & $10(55.56)$ \\
IV & $7(38.89)$ \\
Surgical procedure & \\
Cystoscopic biopsy & $3(16.67)$ \\
TUR-BT & $4(22.22)$ \\
Radical cystectomy & $9(50.00)$ \\
Partial cystectomy & $2(11.11)$ \\
\hline
\end{tabular}

Abbreviation: TUR-BT, transurethral resection of the bladder tumor.

The mean age at diagnosis was 70.67 years (range 59-83 years), and the male-to-female ratio was 2.6:1. Thirteen $(72.22 \%)$ patients gave a history of cigarette smoking. All patients presented with symptoms of gross hematuria, and three of them had bladder tamponade and required blood clot evacuation by cystoscopy. Acute renal failure occurred in two patients who received temporary hemodialysis. None of the 18 patients had paraneoplastic syndrome.

Except for one patient who had T1 disease, all patients presented with either locally advanced disease or metastatic disease when they were initially diagnosed. As shown in Table 1 , ten patients $(55.56 \%)$ had stage III disease, and seven patients $(38.89 \%)$ had lymph node or distant metastasis (stage IV). Treatment varied greatly among patients for whom information was available, and this is presented in Table 2. There were eleven patients who received a major operation (either radical or partial cystectomy). However, very high margin positive rates were noted ( 6 of $11,54.55 \%$ ). Among the patients who received chemotherapy, cisplatin plus etoposide combination and carboplatin/etoposide were the most common regimens.

The tumor characteristics are shown in Table 3. The mean tumor size was $4.29 \mathrm{~cm}(0.5-9.0 \mathrm{~cm})$ in diameter. The intravesical distributions of the tumor were dispersed. The tumor did not occur in any specific location within the bladder. 
Table 2 Treatment modalities of the 18 cases of primary bladder small cell carcinoma

\begin{tabular}{ll}
\hline Treatment modalities & No of patients \\
\hline Radical cystectomy $+C / T+R / T$ & 6 \\
Radical cystectomy $+R / T$ & 3 \\
Partial cystectomy $+C / T+R / T$ & 2 \\
TUR-BT $+C / T+R / T$ & 2 \\
TUR-BT & 1 \\
Cystoscopic biopsy $+C / T+R / T$ & 2 \\
Cystoscopic biopsy & 2 \\
\hline
\end{tabular}

Abbreviations: $C / T$, chemotherapy; R/T, radiotherapy; TUR-BT, transurethra resection of the bladder tumor.

In the majority of patients $(61.11 \%), \mathrm{SCC}$ coexisted with UC components. Only seven patients $(38.89 \%)$ had pure SCC of the bladder.

The mean follow-up time was 19.85 months (range $2-128$ months). Among the eleven patients who were initially metastasis free ( $\mathrm{T} 1$ to $\mathrm{T} 3$ disease), ten patients had tumor recurrence on the extravesical organs. The mean time to recurrence was 8.03 months (range 2.13-25.43 months). As shown in Table 4, a total of 25 recurrent tumors were found in these patients. The most common metastatic organs were the lung and the liver (44.4\%), followed by the bone (27.78\%).

Among the 18 patients who were followed up in our study to date, only three of them survived, and the other 15 had died. These deceased patients survived an average of 10.92 (2-38) months since they were first diagnosed with cancer. The overall survival rates of the patients with bladder SCC and $\mathrm{UC}$ are demonstrated in Figure 1. We can see that patients with bladder SCC had worse overall survival rates than those of stage III and stage IV bladder UC (Log-rank $<0.0001$ ), as well as patients of stage I and II disease (Log-Rank $<0.0001)$. Since most of the patients with bladder SCC presented with stage III and stage IV disease, we further compared their

Table 3 Tumor characteristics

\begin{tabular}{ll}
\hline & Number of patients (\%) \\
\hline Tumor size (diameter) & \\
$<4 \mathrm{~cm}$ & $8(44.44)$ \\
$4-6 \mathrm{~cm}$ & $8(44.44)$ \\
$>6 \mathrm{~cm}$ & $2(11 . \mathrm{II})$ \\
Main tumor location & \\
Dome & $3(16.67)$ \\
Left lateral & $5(27.78)$ \\
Right lateral & $3(16.67)$ \\
Posterior & $7(38.89)$ \\
Tumor components & $7(38.89)$ \\
Pure SCC & $11(61 . I I)$ \\
SCC + UC & \\
\hline
\end{tabular}

Abbreviations: SCC, small cell carcinoma; UC, urothelial carcinoma.
Table 4 Locations of tumor recurrence $(n=25)$

\begin{tabular}{ll}
\hline Location & Number of sites (\%) \\
\hline Lung & $8(44.4)$ \\
Liver & $8(44.4)$ \\
Bone & $5(27.78)$ \\
Central vessel lymph nodes & $2(11.11)$ \\
Brain & $\mathrm{I}(5.56)$ \\
Pelvic cavity & $\mathrm{I}(5.56)$ \\
\hline
\end{tabular}

overall survival rates with those of stage III and stage IV bladder UC, as shown in Figure 2.

Group 1 represented patients with bladder SCC who received radical cystectomy. Group 2 represented patients with bladder SCC who did not receive radical cystectomy. Group 3 represented patients with bladder UC who received radical cystectomy. Group 4 represented patients with bladder UC who did not receive radical cystectomy. We can see that patients with bladder UC who received radical cystectomy had a better overall survival rate than those who did not (Log-Rank <0.0001). On the contrary, in the SCC groups, the overall survival rates were not significantly different whether they received radical cystectomy or not. In other words, performing radical cystectomy for patients with bladder SCC does not significantly improve their overall survival rates. Table 5 shows the possible predictive value for the overall survival rate of bladder SCC patients. None of the clinicopathologic parameters, including the presence of coexisting nonsmall cell carcinoma component $(P=0.831)$, receiving radical cystectomy $(P=0.194)$, distant metastasis $(P=0.062)$, and gender $(P=0.564)$, were significantly associated with survival.

\section{Discussion}

Although primary small cell carcinoma of the urinary bladder is a rare malignant disease and accounts for only less than $1 \%$ of all urinary bladder cancers, a marked increase in the incidence of bladder SCC in the past 15 years was reported. According to Koay et al, there was a significant increase in incidence from 0.05 to 0.14 cases per 100,000 people, and an increase in the percentage of all bladder cancers from 1991 to 2005 (from $0.3 \%$ to $0.6 \%, P<0.0001$ ). ${ }^{9}$ In a chart review of the past 18 years in our institution, there were 18 cases of bladder SCC, which accounted for $0.74 \%$ of all bladder malignancy. Among the 18 patients from our institution, six of the cases occurred between 1995 and 2003, and the other twelve fell between 2004 and 2012. From this data, we found that there was an increasing trend for bladder SCC, which matches Koay et al's findings. 


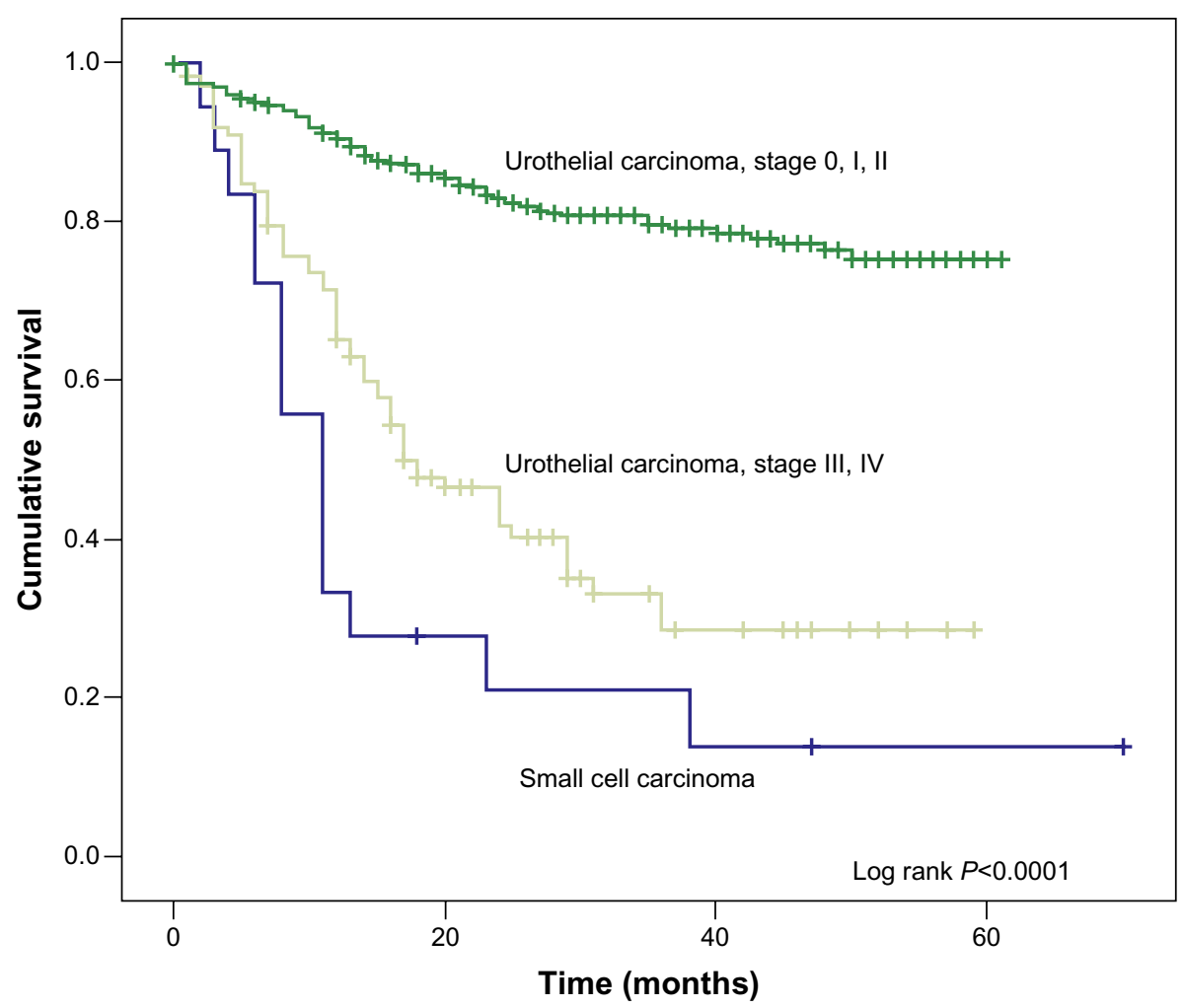

Figure I Kaplan-Meier analysis of the overall survival rates of the patients with bladder SCC and UC. Abbreviations: SCC, small cell carcinoma; UC, urothelial carcinoma.

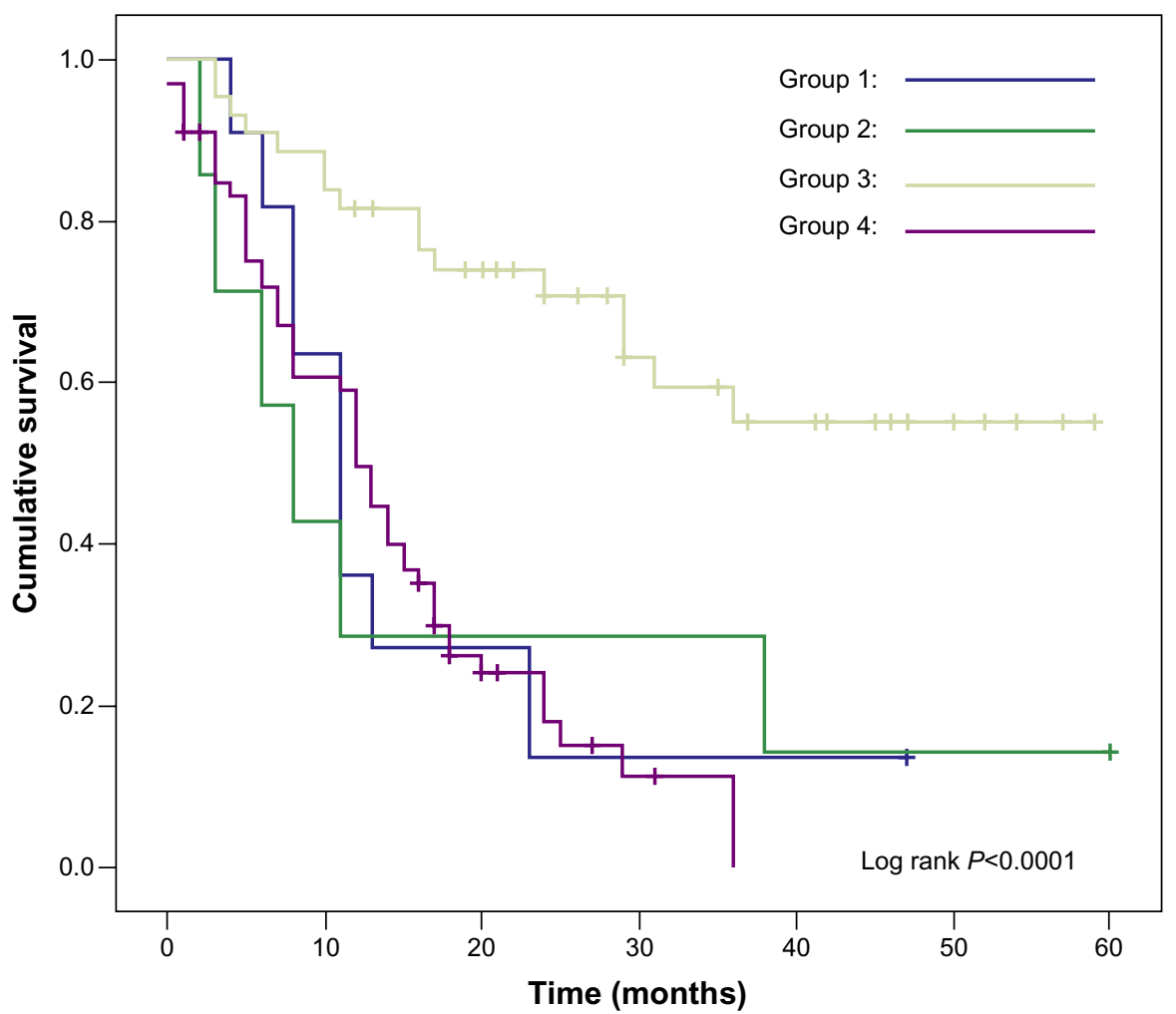

Figure 2 Kaplan-Meier analysis of the overall survival rates with those of stage III and stage IV bladder UC. Note that the Group I represented patients with bladder SCC who received radical cystectomy. Group 2 represented patients with bladder SCC who did not receive radical cystectomy. Group 3 represented patients with bladder UC who received radical cystectomy. Group 4 represented patients with bladder UC who did not receive radical cystectomy.

Abbreviations: SCC, small cell carcinoma; UC, urothelial carcinoma. 
Table 5 Analysis of factors of possible predictive value for the overall survival rate

\begin{tabular}{lll}
\hline & \multicolumn{2}{l}{ Multivariate analysis } \\
\cline { 2 - 3 } & HR (95\% CI) & P-value \\
\hline Pure small cell carcinoma & I.I44 $(0.333-3.927)$ & $0.83 \mathrm{I}$ \\
Radical cystectomy & $2.931(0.578-4.85 \mathrm{I})$ & 0.194 \\
$\mathrm{~N}$ or M stage & $6.273(0.914-7.068)$ & 0.062 \\
Male & $0.658(0.159-2.720)$ & 0.564 \\
\hline
\end{tabular}

Abbreviations: $\mathrm{Cl}$, confidence interval; $\mathrm{HR}$, hazard ratio; $\mathrm{M}$, metastasis; $\mathrm{N}$, node.

The pathogenesis of bladder SCC is still unknown. The possible mechanism may be linked to loss of genetic material, hypermethylation of tumor suppressors, and amplification of the chromosomal regions carrying oncogenes. ${ }^{10}$ Environmental and genetic risk factors in an aging population may also contribute to this disease. ${ }^{11}$ Previous studies postulated that the exposure to tobacco smoke may contribute to SCC. ${ }^{11}$ According to previous literature, most patients were in the sixth to seventh decade of life, males predominated, and many had a history of cigarette smoking. The study by Church et al revealed that cigarette smoking is a risk factor for SCC of the bladder with $50 \%$ to $70 \%$ of patients reporting a smoking history. ${ }^{12}$ The most common presentation was gross hematuria. In our study, this disease is male predominant, with the mean age of 70.67 years, and more than $70 \%$ of the patients had a history of cigarette smoking. All patients presented with symptoms of gross hematuria, and three of them had bladder tamponade requiring blood clot evacuation by cystoscopy. The mean tumor size was $4.29 \mathrm{~cm}$ in diameter, which was far larger than the size of average bladder tumors at the time of discovery. ${ }^{13}$

The diagnosis of bladder SCC is based on the criteria established by the WHO classification system (2004), which was used for the diagnosis of lung SCC. ${ }^{14}$ On light microscopy, the cancer cells stained with haematoxylin and eosin showed packed cells having scant cytoplasm containing few organelles. Tumors were composed of nests of small round malignant cells with pyknotic round to oval nuclei and evenly dispersed "salt and pepper chromatin". ${ }^{14}$ Immunohistochemistry plays an important role in the diagnosis of bladder SCC through the staining of tumor components by antibody markers targeting the following antigens: neuron-specific enolase (NSE), chromogranin, synaptophysin, serotonin, cytokeratin, S-100 protein, thyroid transcription factor 1 (TTF1), epidermal growth factor receptor (EGFR) and C-KIT. ${ }^{15-17}$ SCC may occur in other genitourinary organs; however, the bladder is the most common site for genitourinary extrapulmonary SCC. ${ }^{18}$ Most of the bladder SCC cases were mixed with other histologic types of carcinoma. ${ }^{18}$ In our study, the combination of SCC with UC was the most frequent type, and this accounted for $61.11 \%$ of our patients. Cramer et al proposed that SCC of the urinary bladder originates from metaplastic urothelium. ${ }^{3}$ The frequent association of SCC of the urinary bladder with UC suggests that SCC may originate from a urothelial stem cell rather than from a specific neuroendocrine precursor cell. Other studies suggest that the origin of bladder SCC might be a multi-potential common stem cell, which has the ability to differentiate into various cell types depending on the influence of a specific transformation or progressionrelated gene. These hypotheses might explain the coexistence of bladder SCC with UC, and the heterogeneity of immune staining. ${ }^{19-21}$

Our study revealed that, when the diagnosis was confirmed, most of the bladder SCC patients were staged as advanced disease. Even though some patients were staged as organ confined disease at the beginning, the disease had a strong trend to progress and became metastatic. In our study, the average duration between the time of tumor discovery and the time of metastasis was only 8.03 months. This explains why most treatment results for bladder SCC were unsatisfactory. Because of the rarity of bladder SCC, there was no standard treatment for the disease. According to the literature, treatment options vary greatly. It has been a debated issue whether receiving radical cystectomy provides a better overall survival for bladder SCC patients. In a review reported by the MD Anderson Cancer Center, radical cystectomy was favored, and $52 \%$ of the patients who underwent cystectomy had a better survival. ${ }^{22}$ Furthermore, patients who received neoadjuvant chemotherapy had significantly better survival than those who did not receive it. ${ }^{22}$ However, in a multi-centered review of localized bladder SCC, the efficacy of cystectomy has been questioned. No survival difference was found between patients undergoing surgery and those without surgery (5-year survival was $16 \%$ versus $18 \%$, respectively). ${ }^{23}$ Most of our patients received radical cystectomy, and according to their post-operative conditions, they were either followed with radiation therapy or adjuvant chemotherapy, as shown in Table 2. Two of our patients had already reached critical status when they arrived at our hospital; therefore, they only received biopsy instead of further treatments in order to prove that their tissue sample was cancerous - both died soon after. Nevertheless, our data showed that radical cystectomy did not help improve the overall survival, which is different from bladder UC. As shown in Figure 2, for patients with $>\mathrm{T} 3$ staging, those with 
bladder UC who received radical cystectomy had a much better overall survival in the end. On the contrary, significance was not seen among bladder SCC patients. Platinumetoposide combination chemotherapy has been employed as the main systemic treatment option for bladder SCC. Chemotherapy was usually combined with other therapeutic modalities, especially in patients whose disease was limited to the regional area. Owing to the rarity of this malignancy, no prospective study has been carried out to establish the efficacy and duration of chemotherapy or the relative efficacy of platinum-etoposide versus other chemotherapeutic regimens. ${ }^{24}$ Since the outcomes of treating bladder SCC remain poor, the improvement in survival may rely on the identification of new molecular markers for early diagnosis and novel targeted therapies. Targeted therapy against the c-kit proto-oncogene has been successful for treating gastrointestinal stromal tumors. A significant proportion of SCcs of the urinary bladder express c-kit, suggesting that it may be useful as a therapeutic target in the future.

As shown in Table 5, our study revealed that there was no remarkable predictive value for the overall survival of primary bladder SCC. Although the patients with lymph nodes or distant metastasis ( $\mathrm{N}$ or $\mathrm{M}$ stage) had worse overall survival, the difference was not statistically significant $(P=0.062)$. Cheng et al reviewed the data of 64 patients, and found that none of the clinicopathologic parameters, including age, gender, presenting symptoms, smoking history, the presence of a coexisting nonsmall cell carcinoma component, cystectomy, chemotherapy, or radiation therapy are relative to the patients' overall survival. ${ }^{23}$ It has been a debated issue whether AJCC stage of bladder SCC impacts the overall survival or not. Podesta and True reported that staging was correlated with survival in their review. ${ }^{25}$ However, Mackey and associates did not observe a correlation when their patients were analyzed retrospectively. ${ }^{26}$ Explanations for this finding can be attributed to the small number of current studies on bladder UC, which is also why the finding was not statistically significant. Apart from this, it may also be possible that the categorization of AJCC is for bladder UC instead of bladder SCC. For example, current AJCC staging for bladder cancer classifies any lymph node involvement as stage IV. However, Koay et al observed that patients who had regional lymph node metastasis but no distant metastases had a better overall survival than patients who had distant metastasis, while these were all grouped as stage IV disease. ${ }^{9}$ Therefore, according to Koay's opinion, staging bladder SCC differently from UC should be investigated further. ${ }^{9}$

\section{Conclusion}

Since the incidence of bladder SCC has increased in the past two decades, we should pay more attention to this malignant disease with very poor prognosis. Although this survey was performed in a single medical center, and there were only 18 cases, this is the first reported study to describe primary bladder SCC in a Taiwanese population. All other studies done in East Asia were mostly small-scale case reports, which lack systematic data compilation. Therefore, even though findings from our current study cannot be generalized for the entire Asian population, we still consider the results to be of great significance. Our study demonstrated that primary bladder SCC incidence was male predominant, and presented at a later stage and a worst prognosis than UC. The treatment modalities varied widely in the literature because of the shortage of previous treatment experience. Identification of new molecular markers for early diagnosis and novel targeted therapies may play an important role in the future. Furthermore, prospective study will be needed to elucidate a more effective treatment for bladder SCC.

\section{Disclosure}

The authors report no conflicts of interest in this work.

\section{References}

1. Blomjous CE, Vos W, De Voogt HJ, Van der Valk P, Meijer CJ. Small cell carcinoma of the urinary bladder. A clinicopathologic, morphometric, immunohistochemical, and ultrastructural study of 18 cases. Cancer. 1989;64(6):1347-1357.

2. Lopez JI, Angulo JC, Flores N, Toledo JD. Small cell carcinoma of the urinary bladder. A clinicopathological study of six cases. Br J Urol. 1994;73(1):43-49.

3. Cramer SF, Aikawa M, Cebelin M. Neurosecretory granules in small cell invasive carcinoma of the urinary bladder. Cancer. 1981;47(4): 724-730.

4. Wang X, MacLennan GT, Lopez-Beltran A, Cheng L. Small cell carcinoma of the urinary bladder - histogenesis, genetics, diagnosis, biomarkers, treatment, and prognosis. Appl Immunohistochem Mol Morphol. 2007;15(1):8-18.

5. Sved P, Gomez P, Manoharan M, Civantos F, Soloway MS. Small cell carcinoma of the bladder. BJU Int. 2004;94(1):12-17.

6. Trias I, Algaba F, Condom E, et al. Small cell carcinoma of the urinary bladder. Presentation of 23 cases and review of 134 published cases. Eur Urol. 2001;39(1):85-90.

7. Mostofi FK, Davis CJ, Sesterhenn IA. WHO histologic typing of urinary bladder tumors. Berlin: Springer, 1999.

8. Edge SB, Byrd DR, Compton CC, Fritz AG, Greene FL, Trotti A, editors. AJCC cancer staging manual (7th ed). New York, NY. Springer, 2010.

9. Koay EJ, Teh BS, Paulino AC, Butler EB. A Surveillance, Epidemiology, and End Results analysis of small cell carcinoma of the bladder: epidemiology, prognostic variables, and treatment trends. Cancer. 2011;117(23):5325-5333.

10. Pant-Purohit M, Lopez-Beltran A, Montironi R, MacLennan GT, Cheng L. Small cell carcinoma of the urinary bladder. Histol Histopathol. 2010;25(2):217-221. 
11. Soriano P, Navarro S, Gil M, Llombart-Bosch A. Small-cell carcinoma of the urinary bladder. A clinico-pathological study of ten cases. Virchows Arch. 2004;445(3):292-297.

12. Church DN, Bahl A. Clinical review - small cell carcinoma of the bladder. Cancer Treat Rev. 2006;32(8):588-593.

13. Millán-Rodríguez F, Chéchile-Toniolo G, Salvador-Bayarri J, Palou J, Vicente-Rodríguez J. Multivariate analysis of the prognostic factors of primary superficial bladder cancer. $J$ Urol. 2000;163(1):73-78.

14. Abrahams NA, Moran C, Reyes AO, Siefker-Radtke A, Ayala AG. Small cell carcinoma of the bladder: a contemporary clinicopathological study of 51 cases. Histopathology. 2005;46(1):57-63.

15. Jones TD, Kernek KM, Yang XJ, et al. Thyroid transcription factor 1 expression in small cell carcinoma of the urinary bladder: an immunohistochemical profile of 44 cases. Hum Pathol. 2005;36(7):718-723.

16. Pan CX, Yang XJ, Lopez-Beltran A, et al. c-kit Expression in small cell carcinoma of the urinary bladder: prognostic and therapeutic implications. Mod Pathol. 2005;18(3):320-323.

17. Terada T. Autopsy case of primary small cell carcinoma of the urinary bladder: KIT and PDGFRA expression and mutations. Pathol Int. 2009;59(4):247-250.

18. Mukesh M, Cook N, Hollingdale AE, Ainsworth NL, Russell SG. Small cell carcinoma of the urinary bladder: a 15-year retrospective review of treatment and survival in the Anglian Cancer Network. BJU Int. 2009;103(6):747-752.

19. Terracciano L, Richter J, Tornillo L, et al. Chromosomal imbalances in small cell carcinomas of the urinary bladder. J Pathol. 1999;189(2): $230-235$.
20. Christopher ME, Seftel AD, Sorenson K, Resnick MI. Small cell carcinoma of the genitourinary tract: an immunohistochemical, electron microscopic and clinicopathological study. J Urol. 1991;146(2): $382-388$.

21. van Hoeven KH, Artymyshyn RL. Cytology of small cell carcinoma of the urinary bladder. Diagn Cytopathol. 1996;14(4):292-297.

22. Siefker-Radtke AO, Dinney CP, Abrahams NA, et al. Evidence supporting preoperative chemotherapy for small cell carcinoma of the bladder: a retrospective review of the MD. Anderson cancer experience. J Urol. 2004;172(2):481-484.

23. Cheng L, Pan CX, Yang XJ, et al. Small cell carcinoma of the urinary bladder: a clinicopathologic analysis of 64 patients. Cancer. 2004;101(5):957-962.

24. Pan CX, Zhang H, Lara PN, Cheng L. Small-cell carcinoma of the urinary bladder: diagnosis and management. Expert Rev Anticancer Ther. 2006;6(12):1707-1713.

25. Podesta AH, True LD. Small cell carcinoma of the bladder. Report of five cases with immunohistochemistry and review of the literature with evaluation of prognosis according to stage. Cancer. 1989;64(3): 710-714.

26. Mackey JR, Au HJ, Hugh J, Venner P. Genitourinary small cell carcinoma: determination of clinical and therapeutic factors associated with survival. J Urol. 1998;159(5):1624-1629.
OncoTargets and Therapy

\section{Publish your work in this journal}

OncoTargets and Therapy is an international, peer-reviewed, open access journal focusing on the pathological basis of all cancers, potential targets for therapy and treatment protocols employed to improve the management of cancer patients. The journal also focuses on the impact of management programs and new therapeutic agents and protocols on

\section{Dovepress}

patient perspectives such as quality of life, adherence and satisfaction. The manuscript management system is completely online and includes a very quick and fair peer-review system, which is all easy to use. Visit http://www.dovepress.com/testimonials.php to read real quotes from published authors. 